Supporting Information

\title{
Kinetic Study of Transition Mutations from G-C to A-T Base Pairs in Watson-Crick DNA Base Pairs: Double Proton Transfers
}

Kei Odai and Keisho Umesaki

School of Science and Engineering, Kokushikan University, Setagaya-ku, Tokyo 154-8515, Japan

Figure 6 in the text shows the temporal change in the probability of tautomerization from a G*-T base pair to a G-T* base pair, and the temporal change in the probability of tautomerization from an A-C* base pair to an $A^{*}-C$ base pair. These temporal changes were calculated based on eq 5 in the text. The quantum mechanical transmission coefficient $T_{\mathrm{Q}}(E)$ in the eq 4 is expressed as follows, using the asymmetric Eckert potentials ${ }^{\mathrm{S} 1, \mathrm{~S} 2}$

$$
T_{\mathrm{Q}}(E)=\frac{\cosh (a+b)-\cosh (a-b)}{\cosh (a+b)+\cosh d}
$$

where $E$ represents the energy of the potential, $a, b$ and $d$ denote

$$
a \equiv \frac{2 \pi \sqrt{2 E}}{\hbar \alpha}, b \equiv \frac{2 \pi \sqrt{2(E-\Delta E)}}{\hbar \alpha}, d \equiv \frac{2 \pi \sqrt{2 B-\hbar^{2} \alpha^{2} / 4}}{\hbar \alpha}
$$

in which $B$ is

$$
B \equiv\left(\sqrt{E_{\mathrm{f}}}+\sqrt{E_{\mathrm{f}}-\Delta E}\right)^{2}
$$

Here $\alpha$ in eq $\mathrm{S} 2$ is

$$
\alpha \equiv 2 \pi v_{\mathrm{TS}} \sqrt{\frac{B}{2 E_{\mathrm{f}}\left(E_{\mathrm{f}}-\Delta E\right)}}
$$

where $v_{\mathrm{TS}}$ denotes the absolute value of the imaginary frequency in the transition state. The definitions of $E_{\mathrm{f}}$ and $\Delta E$ in eq $\mathrm{S} 2$ to eq $\mathrm{S} 4$ correspond to Figure 2 in the text. The asymmetric Eckert 
potential has 3 parameters: $E_{\mathrm{f}}, \Delta E$ and $\nu_{\mathrm{TS}}$, and the potential shape is determined by these 3 values. This supporting information shows the values of the parameters for $\mathrm{G}-\mathrm{C}, \mathrm{G}^{*}-\mathrm{T}$, and $\mathrm{A}-\mathrm{C}^{*}$, and the quantum mechanical transmission coefficients for these parameters. For the tunneling factor of G-C, we used the value of Wigner correction $\kappa=3.54$ in the previous paper. ${ }^{\mathrm{S} 3}$ Considering the consistency with $\mathrm{G}^{*}-\mathrm{T}$ and $\mathrm{A}-\mathrm{C}^{*}$, the tunneling factor of G-C should also be evaluated using the asymmetric Eckert potential.

\section{G-C base pair}

Figure S1 shows the potential curve around the transition state of the IRC for G-C. The red curve represents the IRC potential curve. The green curve represents the Eckert potential drawn using the values reported in the previous paper ${ }^{\mathrm{S} 3}$ for the 3 parameter values. The 3 parameter values are $E_{\mathrm{f}}=$ $15.8 \mathrm{kcal} / \mathrm{mol}, \Delta E=10.0 \mathrm{kcal} / \mathrm{mol}, v_{\mathrm{TS}}=998 \mathrm{~cm}^{-1}$. The quantum mechanical transmission coefficient is sensitive to the shape of the top of the potential. Therefore, the blue curve was drawn by adjusting the parameter $v_{\mathrm{TS}}$ so that the Eckert potential fits the shape of the top of IRC. The 3 parameter values for drawing this blue curve were $E_{\mathrm{f}}=15.8 \mathrm{kcal} / \mathrm{mol}, \Delta E=10.0 \mathrm{kcal} / \mathrm{mol}$, and $\nu_{\mathrm{TS}}=1070 \mathrm{~cm}^{-1}$. Thus, we adjusted the parameter $v_{\text {TS }}$ to fit the shape around the top of the IRC.

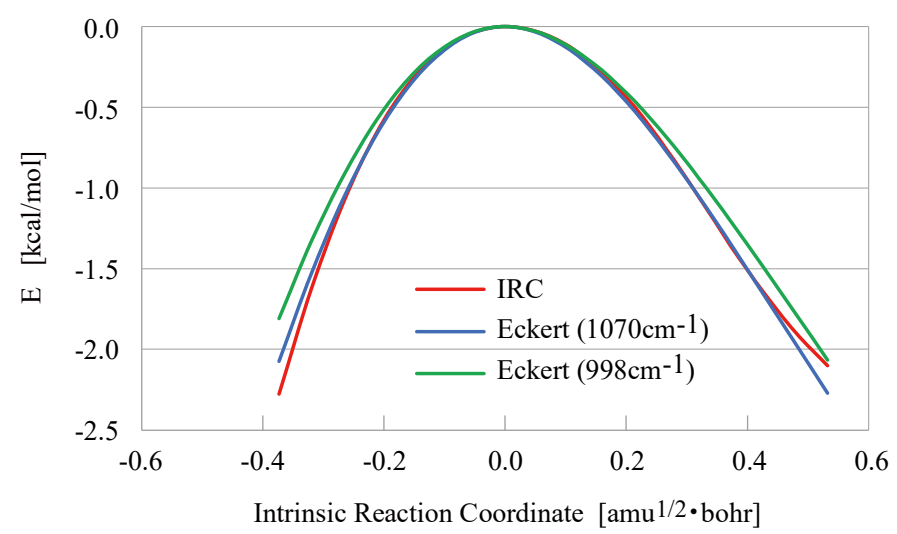

Figure S1. Potential energy curves around the transition state along the IRC of G-C. The red curve 
represents the IRC potential. The blue and green curves represent the Eckert potentials with $v_{\mathrm{TS}}=$ $1070 \mathrm{~cm}^{-1}$ and $v_{\mathrm{TS}}=998 \mathrm{~cm}^{-1}$, respectively.

Figure S2 shows the quantum mechanical transmission coefficient (orange line) and the classical mechanical transmission coefficient (black line) calculated with the parameters of the blue curve in Figure S1. Classically, G-C with an energy greater than the potential peak always changes to $\mathrm{G}^{*}-\mathrm{C}^{*}$. Quantum mechanically, even G-C with energy smaller than the potential peak has a probability of penetrating the potential barrier and changing to $\mathrm{G}^{*}-\mathrm{C}^{*}$. In addition, even $\mathrm{G}-\mathrm{C}$ with energy larger than the potential peak has a probability of not changing to $\mathrm{G}^{*} \mathrm{C}^{*}$. The tunneling factor calculated by eq 4 in the text using the Eckert potential was $\kappa=3.30$. The definite integral was calculated numerically using the Simpson method. The tunneling factor by Wigner correction is $\kappa=3.54$ using $v_{\mathrm{TS}}=$ $998 \mathrm{~cm}^{-1} .{ }^{\mathrm{S} 3}$ The difference between these 2 values is thought to be due to the asymmetry of the IRC potential.

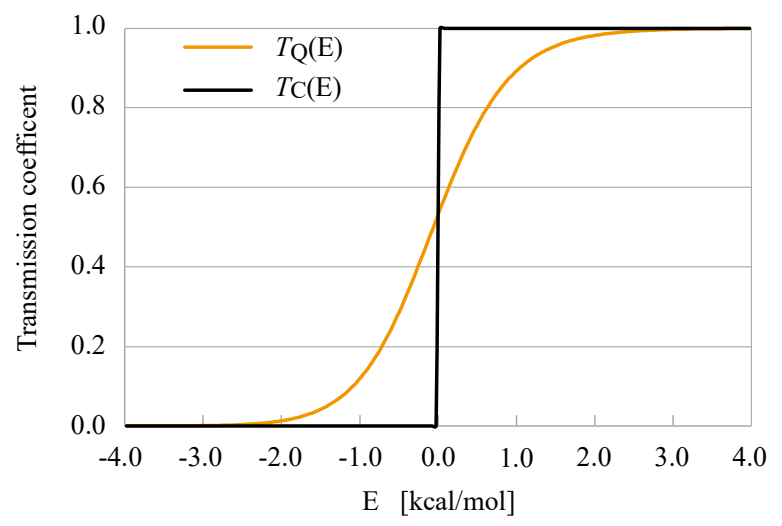

Figure S2. Transmission coefficients for the G-C potential barrier. $T_{\mathrm{Q}}(E)$ and $T_{\mathrm{C}}(E)$ represent the quantum mechanical transmission coefficient and the classical mechanical transmission coefficient, respectively. 


\section{G*-T mismatch pair}

Figure S3 shows the IRC potential for $\mathrm{G}^{*}-\mathrm{T}$ (red) and the parameter-fitted Eckert potential (blue). The 2 parameters $E_{\mathrm{f}}$ and $\Delta E$ are both the same as the values obtained by the IRC calculation, $E_{\mathrm{f}}=$ $6.11 \mathrm{kcal} / \mathrm{mol}, \Delta E=0.898 \mathrm{kcal} / \mathrm{mol} . \quad v_{\mathrm{TS}}$ was adjusted at $1080 \mathrm{~cm}^{-1}$. The imaginary frequency in the IRC calculation was $1173 \mathrm{~cm}^{-1}$. In Figure S3, the shape of the Eckert potential does not fit the IRC potential very well. This is due to the appearance of a shoulder in the IRC potential of $\mathrm{G}^{*}-\mathrm{T}$. With the Eckert potential, it is possible to reproduce the asymmetry of the shape, but it is extremely difficult to reproduce the shape of the shoulder.

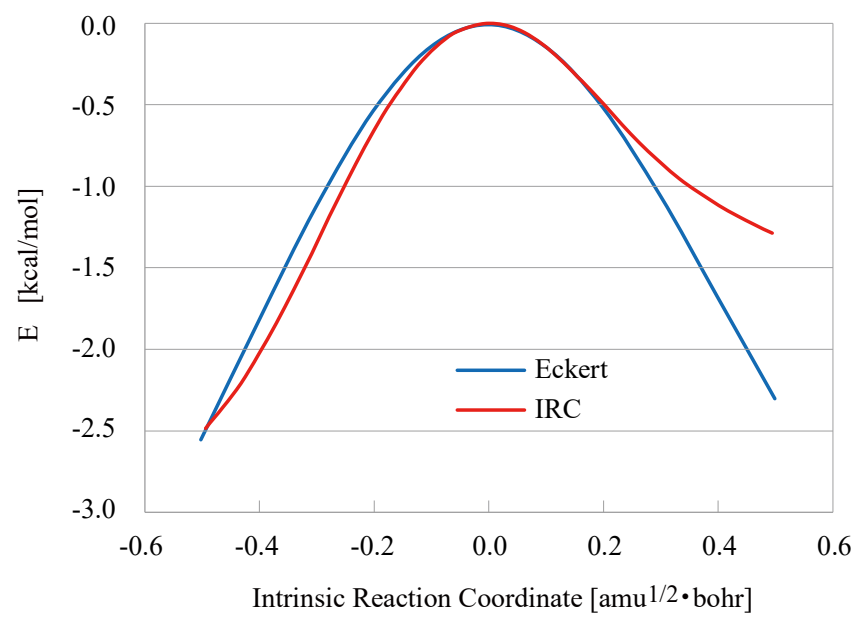

Figure S3. Potential energy curves near the transition state along the IRC of the $\mathrm{G}^{*}-\mathrm{T}$. The red curve represents the IRC potential. The blue curve represents the Eckert potential calculated with 3 parameters, $E_{\mathrm{f}}=6.11 \mathrm{kcal} / \mathrm{mol}, \Delta E=0.898 \mathrm{kcal} / \mathrm{mol}$, and $\nu_{\mathrm{TS}}=1080 \mathrm{~cm}^{-1}$.

Figure S4 shows the quantum mechanical transmission coefficient (orange) and the classical transmission coefficient (black) calculated using the Eckert potential in case of the IRC for G*-T. The tunneling effect appears in the energy range of -1.5 to $1.5 \mathrm{kcal} / \mathrm{mol}$. The tunneling factor calculated by eq 4 in the text was $\kappa=3.33$. 


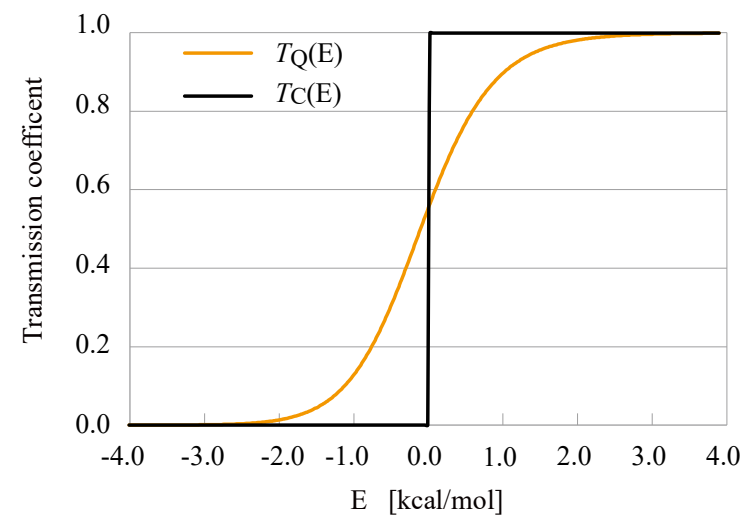

Figure S4. Transmission coefficients for the $\mathrm{G}^{*}-\mathrm{T}$ potential barrier. $T_{\mathrm{Q}}(E)$ and $T_{\mathrm{C}}(E)$ represent the quantum mechanical transmission coefficient and the classical mechanical transmission coefficient, respectively.

\section{A-C* mismatch pair}

Figure S5 shows the IRC potential for A-C* (red) and the parameter-fitted Eckert potential (blue). The 2 parameters $E_{\mathrm{f}}$ and $\Delta E$ are both the same as the values obtained by the IRC calculation, $E_{f}=$ $10.5 \mathrm{kcal} / \mathrm{mol}, \Delta E=3.26 \mathrm{kcal} / \mathrm{mol} . v_{\mathrm{TS}}$ was adjusted at $650 \mathrm{~cm}^{-1}$. The imaginary frequency in the IRC calculation was $936 \mathrm{~cm}^{-1}$. In Figure S5, the shape of the Eckert potential does not fit the IRC potential very well. This is because, like $\mathrm{G}^{*}$-T, the IRC potential of A-C* has a shoulder.

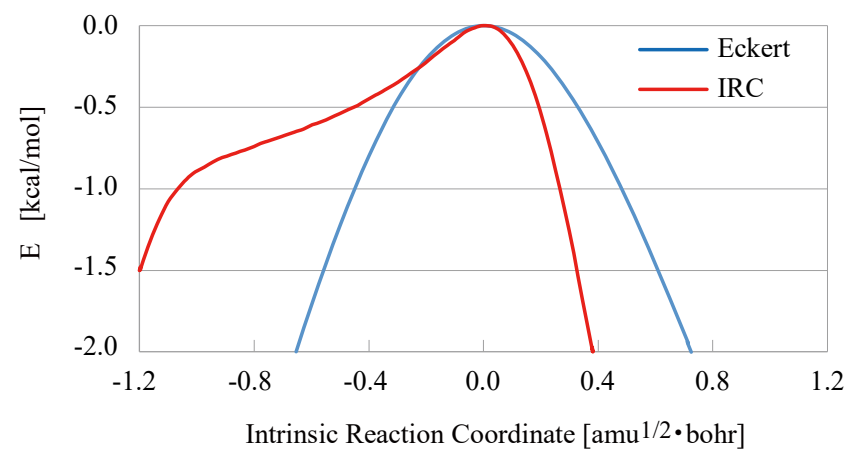

Figure S5. Potential energy curves near the transition state along the IRC of the A-C*. The red curve represents the IRC potential. The blue curve represents the Eckert potential calculated with 3 
parameters, $E_{\mathrm{f}}=10.5 \mathrm{kcal} / \mathrm{mol}, \Delta E=3.26 \mathrm{kcal} / \mathrm{mol}$, and $v_{\mathrm{TS}}=650 \mathrm{~cm}^{-1}$.

Figure S6 shows the quantum mechanical transmission coefficient (orange) and the classical transmission coefficient (black) calculated using the Eckert potential in case of the IRC for A-C*. The tunneling effect appears in the energy range of -1.0 to $1.0 \mathrm{kcal} / \mathrm{mol}$. The tunneling factor calculated by eq 4 in the text was $\kappa=1.56$.

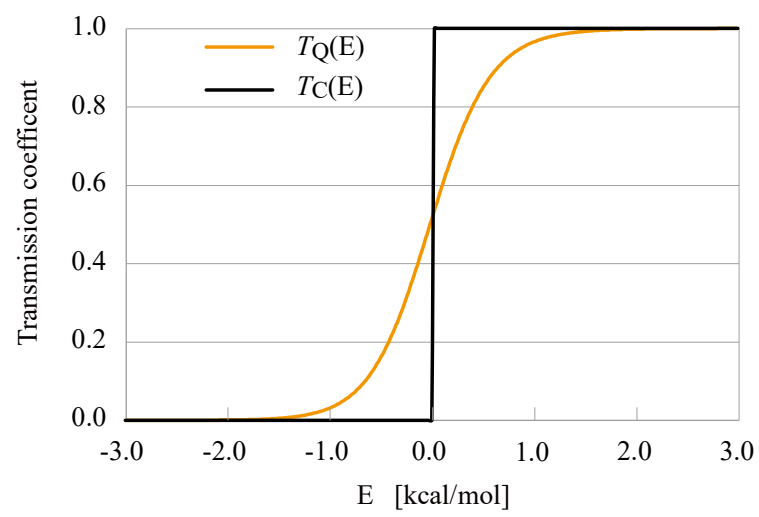

Figure S6. Transmission coefficients for the A-C* potential barrier. $T_{\mathrm{Q}}(E)$ and $T_{\mathrm{C}}(E)$ represent the quantum mechanical transmission coefficient and the classical mechanical transmission coefficient, respectively.

\section{REFERENCES}

(S1) Eckert, C. The penetration of a potential barrier by electrons. Phys. Rev., 1930, 35, 1303-1309.

(S2) Bao, J. L.; Truhlar, D. G. Variational transition state theory: theoretical framework and recent developments. Chem. Soc. Rev., 2017, 46, 7548-7596.

(S3) Umesaki, K.; Odai, K. A Kinetic Approach to Double Proton Transfer in Watson-Crick DNA Base Pairs. J. Phys. Chem. B., 2020, 124, 1715-1722. 